\title{
Geometrical Bounds on Irreversibility in Open Quantum Systems
}

\author{
Luca Mancino, ${ }^{1, *}$ Vasco Cavina, ${ }^{2}$ Antonella De Pasquale, ${ }^{3,4,2}$ Marco Sbroscia, ${ }^{1}$ Robert I. Booth,,${ }^{5,1}$ \\ Emanuele Roccia, ${ }^{1}$ Ilaria Gianani, ${ }^{1}$ Vittorio Giovannetti, ${ }^{2}$ and Marco Barbieri ${ }^{1,6}$ \\ ${ }^{1}$ Dipartimento di Scienze, Università degli Studi Roma Tre, Via della Vasca Navale 84, 00146, Rome, Italy \\ ${ }^{2}$ NEST, Scuola Normale Superiore and Istituto Nanoscienze-CNR, Piazza dei Cavalieri 7, I-56126, Pisa, Italy \\ ${ }^{3}$ Dipartimento di Fisica, Università di Firenze, Via G. Sansone 1, I-50019 Sesto Fiorentino (FI), Italy \\ ${ }^{4}$ INFN Sezione di Firenze, via G.Sansone 1, I-50019 Sesto Fiorentino (FI), Italy \\ ${ }^{5}$ Institut de Physique, Sorbonne Université, 4 Place Jussieu, 75005, Paris, France \\ ${ }^{6}$ Istituto Nazionale di Ottica-CNR, Largo Enrico Fermi 6, 50125, Florence, Italy
}

(Received 22 January 2018; revised manuscript received 1 August 2018; published 17 October 2018)

\begin{abstract}
The Clausius inequality has deep implications for reversibility and the arrow of time. Quantum theory is able to extend this result for closed systems by inspecting the trajectory of the density matrix on its manifold. Here we show that this approach can provide an upper and lower bound to the irreversible entropy production for open quantum systems as well. These provide insights on how the information on the initial state is forgotten through a thermalization process. Limits of the applicability of our bounds are discussed and demonstrated in a quantum photonic simulator.
\end{abstract}

DOI: 10.1103/PhysRevLett.121.160602

Introduction.-Irreversibility in physical processes is strictly related to the idea of energy dissipation. This concept is one of the cornerstones of thermodynamics since it came to the stage in the second half of the 19th century. The second law of thermodynamics allows us to give a quantitative characterization to the interplay between the irreversibility and the exchange of energy, introducing a state function, the entropy, that is always nondecreasing in macroscopical processes [1]. The generality of this principle is a key feature in establishing thermodynamics as one of the fundamental branches of classical theories and plays a role also in the connections between physics and information science [2-4]. A better understanding of the implication of the second law at the quantum level is one of the main issues of the actual research in the field of quantum thermodynamics [5-10] and is fundamental in the construction of a solid theoretical ground for a wide class of applications such as computation [11], metrology [12-15], quantum control [16-18], and quantum thermal engines [19-21].

In this Letter, we examine the thermalization process of a quantum system $\mathcal{A}$ in contact with a reservoir, as pictorially represented in Fig. 1. For this significant example of out-ofequilibrium evolution, we manage to bound the irreversible entropy production both from above and below, facing the problem within an informational-geometric setting [22,23]. While the upper bound obtained here is completely general, the lower bound holds only under certain conditions, satisfied, for example, in the single-qubit scenario.

We will treat the Hilbert space of the system $\mathcal{A}$ as a Riemannian manifold. This allows us to establish relations between the irreversible entropy production and the geodesic distances corresponding to metrics that are contractive under complete positive and trace preserving maps. Following a similar approach, S. Deffner and E. Lutz [24] determined a lower bound to the irreversible entropy production for a closed quantum system driven by an external field, in terms of the Bures length. However, it results that the latter is not the only contractive Riemannian metric on the system Hilbert space, as there exists an infinite family of such metrics as characterized by the Morozova-Čencov-Petz theorem [25,26]. Within this "mare magnum," the only Riemannian metrics whose geodesics are analytically known are the Bures and the WignerYanase one. Quite recently, Pires et al. [27] proved that the Bures metrics for the case of any single-qubit unitary dynamics effectively provides a tighter bound on the transformation speed at least when compared to the Wigner-Yanase one; these transformations encompass those considered in [24]. In general, establishing which

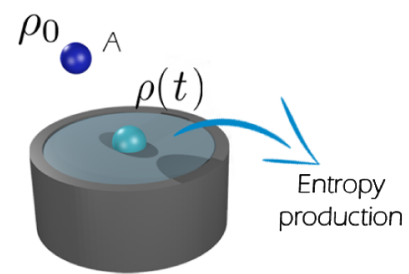

(a) HEAT BATH

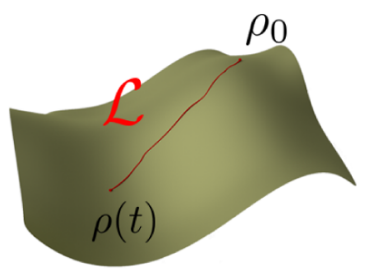

(b) RIEMANNIAN MANIFOLD
FIG. 1. Principal features of our scheme: (a) the quantum system is initialized in a given state $\rho_{0}$, then interacts with a reservoir at temperature $T$. The evolution induced by the interactions brings the system in a state $\rho(t)$ and produces irreversible entropy. (b) Studying the trajectory on the state space allows for information-geometric considerations delivering a lower bound on the produced entropy in terms of the geodesic length $\mathcal{L}$. 
metrics returns the smallest geodesic distance between states is still an open question [27,28]. In our Letter, we compare the two above mentioned metrics for the case of a qubit thermalization process described by the so-called generalized amplitude damping map and show that in this case the Wigner-Yanase one provides the sharper bounds to the irreversible entropy production. Our results are supported by experimental evidence, gained by simulating the thermalization of a qubit in contact with a bosonic bath, using quantum photonics $[29,30]$. We have compared the performances of two states: (i) the quantum superposition state of the ground and the excited levels of $\mathcal{A}$ and (ii) the ground state of $\mathcal{A}$ : we have found that only the former is able to efficiently distinguish the performances of the two metrics. On a more practical ground, our bounds can be simply computed by performing the tomography of the state of the system at fixed time instants. In some cases, this represents a less demanding task than directly measuring thermodynamic functionals due to the nonlinear expressions of the latter.

Geometrical bounds for irreversible entropy production.-Let us consider an arbitrary system $\mathcal{A}$ weakly coupled with a thermal bath at temperature $T$. Without loss of generality, let us fix as $t=0$ the initial time $t$ of the process, and let $\rho_{0}$ be the associated density matrix of the system. If we keep the system Hamiltonian $H$ unchanged, the system will thermalize with the reservoir, thus asymptotically reaching the canonical equilibrium state $\rho_{\mathrm{eq}}=e^{-\beta H} / \mathcal{Z}_{\beta}$, where $\beta=1 / k_{B} T, k_{B}=1$ is the Boltzmann constant, and $\mathcal{Z}_{\beta}$ is the partition function. This represents a prototypical example of an irreversible thermodynamic evolution, which, for instance, finds applications also in the context of quantum thermometry [12-14,29-32]. From a mathematical point of view, this process can be described by a two-parameters family of quantum channels $\left\{\Phi_{t}^{T}\right\}$, such that for all $t$ associates the density matrix $\rho(t):=\Phi_{t}^{T}\left[\rho_{0}\right]$ of the system to the initial state $\rho_{0}$. This map admits a unique stationary state corresponding to the (unique) equilibrium state of the map, i.e., $\Phi_{t}^{T}\left(\rho_{\text {eq }}\right)=\rho_{\text {eq }}$. The thermodynamically irreversible component of the entropy variation $\Delta S_{\mathcal{A}}^{\text {irr }}$ produced due to evolution from $t=0$ to a generic time $t$ is given by

$$
\Delta S_{\mathcal{A}}^{\mathrm{irr}}:=\Delta S_{\mathcal{A}}-\frac{\Delta Q}{T},
$$

where the first term on the right-hand side is the entropy variation of the system $\Delta S_{\mathcal{A}}=S_{\mathcal{A}}[\rho(t)]-S_{\mathcal{A}}\left(\rho_{0}\right)$, being $S_{\mathcal{A}}(\rho)=-\operatorname{Tr}(\rho \ln \rho)$ the von Neumann entropy of the density matrix $\rho$, and the second term is the heat $\Delta Q=$ $\operatorname{Tr}[H \rho(t)]-\operatorname{Tr}\left[H \rho_{0}\right]$ absorbed by the system and corresponds to the thermodynamically reversible contribution to the entropy production. The Clausius inequality, $\Delta S_{\mathcal{A}}^{\mathrm{irr}} \geq 0$, provides a process-independent lower bound to $\Delta S_{\mathcal{A}}^{\text {irr }}$. In this Letter, we will write both a sharper lower bound and also an upper bound to this thermodynamic functional, by recasting it as [33-35]

$$
\Delta S_{\mathcal{A}}^{\mathrm{irr}}(t)=S\left(\rho_{0} \| \rho_{\mathrm{eq}}\right)-S\left(\rho(t) \| \rho_{\mathrm{eq}}\right),
$$

where $S\left(\rho_{1} \| \rho_{2}\right)=\operatorname{Tr}\left(\rho_{1} \ln \rho_{1}\right)-\operatorname{Tr}\left(\rho_{1} \ln \rho_{2}\right)$ is the socalled relative entropy of $\rho_{1}$ to $\rho_{2}$. Notice that in this case the Clausius inequality can be easily proved from (2), by exploiting the monotonicity of the relative entropy under quantum channels, i.e., completely positive trace preserving operations. Our interest for this relation stems from an inequality of geometrical nature that links the quantum relative entropy with the unitarily invariant norms on the Hilbert space. More precisely, calling $\mathcal{L}$ the distance induced on the Hilbert space by a given unitarily invariant norm, we have $S(\rho \| \sigma) \geq 2 \mathcal{L}^{2}(\rho, \sigma) / \mathcal{L}^{2}\left(e_{1,1}, e_{2,2}\right)$, where $e_{i, j}$ is the matrix, with the $i, j$ element equal to 1 and all other elements 0 [36]. We require the distance $\mathcal{L}$ to be contractive under the action of any map $\Lambda$, i.e., $\mathcal{L}(\Lambda(\rho), \Lambda(\sigma)) \leq \mathcal{L}(\rho, \sigma)$. The latter condition is crucial in order to interpret $\mathcal{L}^{2}$ as a measure of the distinguishability between $\rho$ and $\sigma$. This is satisfied by an infinite family of metrics explicitly characterized by the Morozova-Čencov-Petz theorem [25,26,37]. It follows that each of such metrics will provide a different consistent bound to the relative entropy. The only cases in which an analytical expression for the geodesic distance is known [27,28] are the quantum Fisher information metric $\mathcal{L}_{\mathrm{QF}}(\rho, \sigma)=$ $\arccos [\operatorname{Tr}[\sqrt{\sqrt{\rho} \sigma \sqrt{\rho}}]]$ and the Wigner-Yanase metric $\mathcal{L}_{\mathrm{WY}}(\rho, \sigma)=\arccos [\operatorname{Tr}[\sqrt{\rho} \sqrt{\sigma}]]$. Notice that, if $\rho$ and $\sigma$ commute, the two geodesic distances coincide. We can use these properties to obtain a sharper geometrical bound to the irreversible component of the entropy during a thermalization process as

$$
\Delta S_{\mathcal{A}}^{\mathrm{irr}}(t) \leq S\left(\rho_{0} \| \rho_{\mathrm{eq}}\right)-\frac{8}{\pi^{2}} \max _{\{X=\mathrm{QF}, \mathrm{WY}\}} \mathcal{L}_{X}^{2}\left(\rho(t), \rho_{\mathrm{eq}}\right),
$$

where we exploited that $\mathcal{L}_{X}^{2}\left(e_{1,1}, e_{2,2}\right)=\pi^{2} / 4, X=\mathrm{QF}$, WY. This is the upper bound to $\Delta S_{\mathcal{A}}^{\text {irr }}$ we are looking for: the geometrical distance establishes how much entropy is dissipated during a generic transformation of a quantum open system. Notice that asymptotically, i.e., for $t \rightarrow \infty$, the inequality above becomes trivially strict, as both members reduce to $S\left(\rho_{0} \| \rho_{\text {eq }}\right)$ [see expression (2)].

The same technique can be exploited to determine a lower bound for $\Delta S_{\mathcal{A}}^{\text {irr }}$. However, in this case, an additional step that restricts our analysis to a specific class of dynamical maps is required. In the specific, we say that a dynamical map $\Phi_{t}$ satisfies a (reverse) triangle inequality for the relative entropy if, taken an initial state $\rho_{0}$ and two instants of time $t_{1}$ and $t_{2} \geq t_{1}$, we have

$S\left[\rho_{0} \| \Phi_{t_{2}}\left(\rho_{0}\right)\right] \geq S\left[\rho_{0} \| \Phi_{t_{1}}\left(\rho_{0}\right)\right]+S\left[\Phi_{t_{1}}\left(\rho_{0}\right) \| \Phi_{t_{2}}\left(\rho_{0}\right)\right]$.

Let us remark that the relation above is not valid, in general. For instance, it can be shown to hold when $\Phi_{t}\left(\rho_{0}\right)=\left(1-\lambda_{t}\right) \rho_{0}+\lambda_{t} \rho_{\text {eq }}, \lambda_{t}$ being an increasing function of time $t$ (see the Supplemental Material [38], 


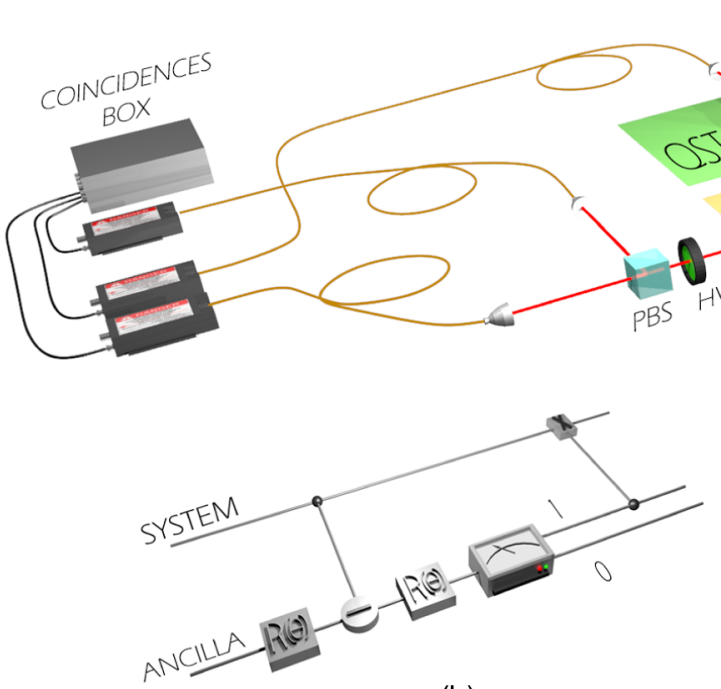

(b)

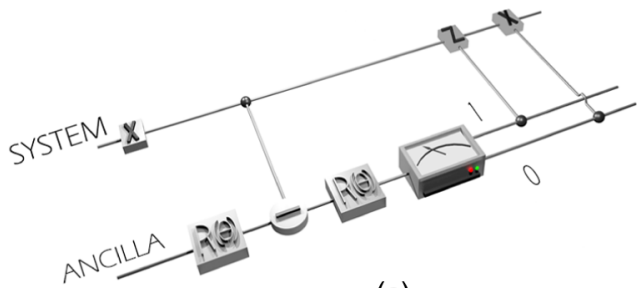

(c)

FIG. 2. Quantum simulation via quantum logic gates. (a) Experimental apparatus. The system and the ancilla photons are generated via a spontaneous parametric down-conversion (SPDC) process through a $\beta$ barium-borate (BBO) crystal pumped with a cw laser (80 mW at $405 \mathrm{~nm}$ ). The SPDC is type I, frequency degenerate (bandwidth of the parametric fluorescence greater than $30 \mathrm{~nm}$ ). To ensure indistinguishability of the photons, these are spectrally filtered to a narrow bandwidth $(7.5 \mathrm{~nm})$ and selected by single-mode fibers. The qubits are injected in the photonic setup which performs the logical operations depicted in (b) and (c) representing, respectively, an amplitude damping (AD) and an inverse amplitude damping (IAD) channel. Their combination allows for reconstructing a generalized amplitude damping (GAD) channel, simulating the thermalization dynamics of the qubit illustrated in the main text [29]. The measured nonclassical interference visibility is 0.78 , to be compared with the expected value 0.80 . List of abbreviations: polarizing beam splitter (PBS), partially polarizing beam splitter (PPBS), half wave plate (HWP), quarter wave plate (QWP), quantum state tomography (QST).

Appendix A). A less trivial case is provided by the generalized amplitude damping channel for qubit systems, specifically considered in the following part of the manuscript. However, if (4) is satisfied by the dynamical process $\Phi_{t}^{T}$ and we choose $t_{1}=t$ and $t_{2}=\infty$, we find

$$
\Delta S_{\mathcal{A}}^{\mathrm{irr}}(t) \geq \frac{8}{\pi^{2}} \max _{\{X=\mathrm{QF}, \mathrm{WY}\}} \mathcal{L}_{X}^{2}\left(\rho_{0}, \rho(t)\right)
$$

Equation (5) represents a tighter version of the Clausius inequality and generalizes to the open system framework the results obtained in [24].

From now on, we will focus on the specific case in which the reference system is a qubit with Hamiltonian $H=\sigma_{z} / 2$ [40] in thermal contact with a bosonic reservoir. Its evolution can be formally described in terms of the GAD channel, $\rho(t)=\Phi_{t}^{T}\left(\rho_{0}\right)$, with Kraus operators [39]: $E_{1}=\sqrt{p}\left(|0\rangle\left\langle 0\left|+\sqrt{1-\eta_{t}}\right| 1\right\rangle\langle 1|\right), \quad E_{2}=$ $\sqrt{p} \sqrt{\eta_{t}}|0\rangle\langle 1|, \quad E_{3}=\sqrt{1-p}\left(\sqrt{1-\eta_{t}}|0\rangle\langle 0|+| 1\rangle\langle 1|\right)$, $E_{4}=\sqrt{1-p} \sqrt{\eta_{t}}|1\rangle\langle 0|$, in the canonical basis $\{|0\rangle,|1\rangle\}$ corresponding, respectively, to the excited and the ground state of the system. Here $p \in[0,1 / 2]$ and $\eta_{t} \in[0,1]$ are the time- and temperature-dependent probability and damping coefficient, respectively, related to the average boson occupation number $\bar{N}=1 /\left(e^{1 / T}-1\right)$ [40] for the bath and to the (dimensionless) time $t$ as $(1-2 p)=$ $(1+2 \bar{N})^{-1}$ and $\eta_{t}=1-e^{-(1+2 \bar{N}) t}[29,31]$. As anticipated above, such thermalizing map can be shown to satisfy the triangle inequality (4); thus, the geometrical lower bound (5) can be enforced [38]. Notice that the GAD map can be realized by combing an IAD map described by $E_{1}$ and $E_{2}$ with an $\mathrm{AD}$ map described by $E_{3}$ and $E_{4}$. The extension to a GAD channel in which the energy gap $\Delta(t)$ between the two energetic levels is externally modulated is discussed in the Supplemental Material [38] (Appendices B and C).

Quantum photonic simulation.-We have simulated the thermalization dynamics of a qubit via a GAD channel to test inequalities (3) and (5). Our experiment is based on a quantum photonics logic gate, as a method to implement general quantum channels [41,42] (other approaches still in the framework of quantum photonics can be found in $[43,44])$. Our setup is shown in Fig. 2(a) and allows us to realize the following steps. (i) The canonical basis is encoded in the horizontal and vertical polarization states, i.e., $|0\rangle \equiv|H\rangle$ and $|1\rangle \equiv|V\rangle$. An ancilla qubit $\mathcal{B}$ is initialized in $|H\rangle$, while the target qubit $\mathcal{A}$ is prepared in a linear polarization state. (ii) The qubits $\mathcal{A}$ and $\mathcal{B}$ interact through a controlled-sign (C-Z) gate, acquiring a $\pi$ phase on the $|V\rangle_{\mathcal{A}}|V\rangle_{\mathcal{B}}$ component only [45-50]; $\mathcal{B}$ undergoes the same rotation $R(\theta)$ before and after the $\mathrm{C}-\mathrm{Z}$, transmitted by HWPs set at an angle $\theta$ : this controls the damping rate as $\eta=\sin ^{2}(4 \theta)$. (iii) The $H / V$ component of $\mathcal{B}$ is measured and the outcomes control a Pauli transformation on $\mathcal{A}$. The arrangements in Figs. 2(b) and 2(c) implement the AD and 

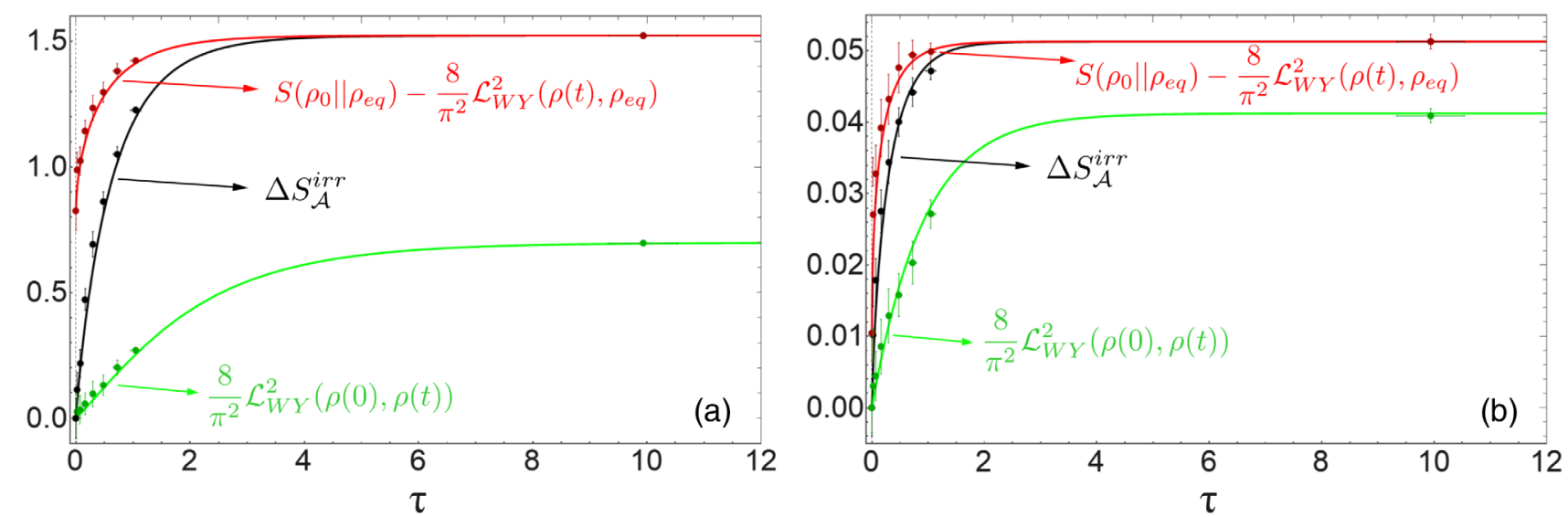

FIG. 3. Lower (green line) and upper (red line) bounds to the irreversible entropy production for a GAD channel induced by a bosonic reservoir, as a function of time $\tau$. The initial state is $|D\rangle=1 / \sqrt{2}(|H\rangle+|V\rangle)$ in (a) and $|V\rangle$ in (b). The solid lines are theoretical predictions; experimental points have been obtained from the density matrices retrieved through quantum state tomography. Vertical errors have been obtained through a Monte Carlo routine that takes into account the Poissonian statistics of the measured counts, while horizontal errors depend on the uncertainty on the rotation $R(\theta)$ [29].

IAD maps, respectively. (iv) The density matrix $\rho(t)$ of the target $\mathcal{A}$ at time $t$ is determined collecting coincidence counts for the quantum state tomography in the two configurations, including the controlled-Pauli operations in postprocessing. We then run the state reconstruction algorithm on data weighted with $p$ and $1-p$ [29]. Examples of the reconstructed states are reported in the Supplemental Material [38], Appendix D.

From the knowledge of the full density matrix of the target qubit, we have been able to determine the relevant quantities in the bounds (3) and (5), reported in Fig. 3 for $T=0.34$. It can be numerically proved that the WignerYanase metric, compared to the Bures one, provides a

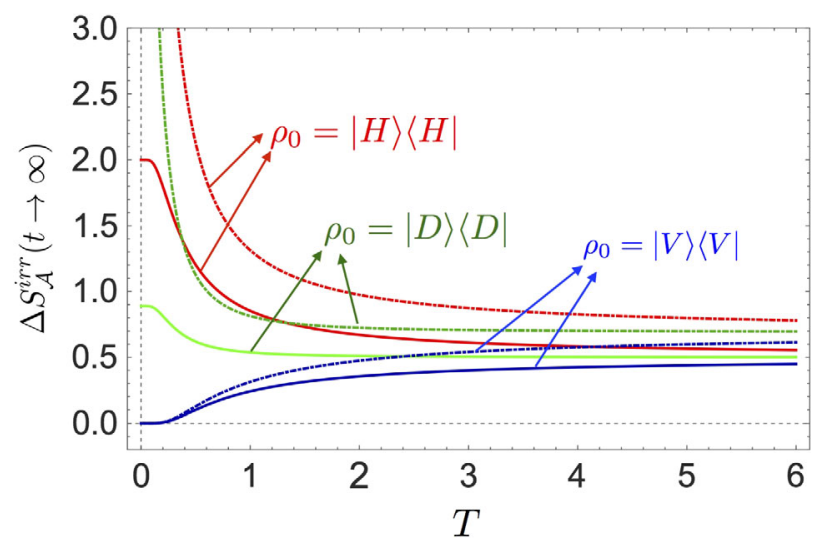

FIG. 4. Asymptotic irreversible entropy production as a function of the temperature $T$ for the thermalization of the system $\mathcal{A}$. The dotted curves show the predictions associated to different pure states $|H\rangle$ (red), $|D\rangle$ (green) and $|V\rangle$ (blue). The solid curves show the geometric lower bound (5) in the thermalization limit for the same three cases, with the same color code, which provides a quite a good prediction for $\Delta S_{\mathcal{A}}^{\mathrm{irr}}(t \rightarrow \infty)$ for almost all values of $T$. sharper bound to the irreversible entropy, for arbitrary choices of the initial state $\rho_{0}$ (the two metrics give the same result when $\rho_{0}$ is diagonal in the chosen basis). An experimental test for the state $|D\rangle=1 / \sqrt{2}(|H\rangle+|V\rangle)$, analyzed in Fig. 3, is provided in the Supplemental Material [38]. For this reason, in Fig. 4 we report the experimental points associated with the Wigner-Yanase metric. The upper bound captures more closely the behavior of $\Delta S_{\mathcal{A}}^{\text {irr }}$. Data closely follow the predictions with a small systematic overestimation of the entropies. The fidelity of the simulated states with the ideal ones are $F_{H}=0.996 \pm 0.003, \quad F_{D}=0.995 \pm 0.005, \quad$ and $F_{V}=$ $0.998 \pm 0.002$ for the three inputs, averaged over all simulated times.

In the limit $t \rightarrow \infty$, the bound (5) gives insights on the entropy produced for losing the initial information on qubit $\mathcal{A}$ by thermalization [51,52]. Our generalization allows us to distinguish specific instances: the theoretical curves in Fig. 4 indicate that different entropies are dissipated for distinct initial pure states, and the lower bound (5) reflects such behavior. Our results confirm that the excited state $|H\rangle$ asymptotically demands more entropy dispersion to be forgotten via thermalization.

Conclusions.-We have shown that the entropy production in open quantum systems can be bounded, both from above and below, with quantities that depend on purely geometrical features of the Hilbert space. Such bounds have been retrieved by explicitly comparing the quantum Fisher metrics with the Wigner-Yanase one, representing the only two examples for which an analytic expression of the associated geodesic length is known. According to our analysis, supported by experimental evidence provided in a quantum optics experiment, the Wigner-Yanase metrics yields the tighter bound. Finally, this study, based on the comparison between different metrics on the Hilbert space 
of the system, shows explicit connections with the computation of the geometrical quantum speed limits [27], thus offering an interesting connection between entropy and time in irreversible physical phenomena.

We are grateful to A. Mari, M. A. Ciampini, R. Raimondi, and M. Paternostro for insightful feedbacks on the manuscript. A.D. P. acknowledges the financial support from the University of Florence in the framework of the University Strategic Project Program 2015 (Project BRS00215).

*luca.mancino@uniroma3.it

[1] R. Clausius, The Mechanical Theory of Heat: With Its Applications to the Steam-Engine and to the Physical Properties of Bodies (J. van Voorst, London, 1867).

[2] R. Landauer, Irreversibility and heat generation in the computing process, IBM J. Res. Dev. 5, 183 (1961).

[3] M. B. Plenio and V. Vitelli, The physics of forgetting: Landauer's erasure principle and information theory, Contemp. Phys. 42, 25 (2001).

[4] J. Goold, M. Huber, A. Riera, L. del Rio, and P. Skrzypczyk, The role of quantum information in thermodynamics-a topical review, J. Phys. A 49, 143001 (2016).

[5] P. Ćwiklinski, M. Studziński, M. Horodecki, and J. Oppenheim, Limitations on the Evolution of Quantum Coherences: Towards Fully Quantum Second Laws of Thermodynamics, Phys. Rev. Lett. 115, 210403 (2015).

[6] M. Horodecki and J. Oppenheim, Fundamental limitations for quantum and nanoscale thermodynamics, Nat. Commun. 4, 2059 (2013).

[7] M. Lostaglio, D. Jennings, and Terry Rudolph, Description of quantum coherence in thermodynamic processes requires constraints beyond free energy, Nat. Commun. 6, 6383 (2015).

[8] S. Vinjanampathy and J. Anders, Quantum thermodynamics, Contemp. Phys. 57, 545 (2016).

[9] G. Francica, J. Goold, and F. Plastina, The role of coherence in the non-equilibrium thermodynamics of quantum systems, arXiv:1707.06950.

[10] J. P. Santos, L. C. Celeri, G. T. Landi, and M. Paternostro, The role of quantum coherence in non-equilibrium entropy production, arXiv:1707.08946.

[11] C. H. Bennett, Notes on Landauer's principle, reversible computation, and Maxwell's Demon, Stud. Hist. Philos. Sci., Part B 34, 501 (2003).

[12] L. A. Correa, M. Mehboudi, G. Adesso, and A. Sanpera, Individual Quantum Probes for Optimal Thermometry, Phys. Rev. Lett. 114, 220405 (2015).

[13] G. De Palma, A. De Pasquale, and V. Giovannetti, Universal local quantum thermal susceptibility, Phys. Rev. A 95, 052115 (2017).

[14] A. De Pasquale, K. Yuasa, and V. Giovannetti, Estimating temperature via sequential measurements, Phys. Rev. A 96, 012316 (2017).

[15] M. Mehboudi, M. Moreno-Cardoner, G. De Chiara, and A. Sanpera, Thermometry precision in strongly correlated ultracold lattice gases, New J. Phys. 17, 055020 (2015).
[16] T. Feldmann and R. Kosloff, Minimal temperature of quantum refrigerators, Europhys. Lett. 89, 20004 (2010).

[17] V. Cavina, A. Mari, and V. Giovannetti, Optimal thermodynamic control in open quantum systems, Phys. Rev. A 98, 012139 (2018).

[18] V. Cavina, A. Mari, and V. Giovannetti, Optimal processes for probabilistic work extraction beyond the second law, Sci. Rep. 6, 29282 (2016).

[19] V. Cavina, A. Mari, and V. Giovannetti, Slow Dynamics and Thermodynamics of Open Quantum Systems, Phys. Rev. Lett. 119, 050601 (2017).

[20] A. del Campo, J. Goold, and M. Paternostro, More bang for your buck: Super-adiabatic quantum engines, Sci. Rep. 4, 6208 (2014).

[21] R. Alicki, The quantum open system as a model of the heat engine, J. Phys. A 12, L103 (1979).

[22] G. Ruppeiner, Thermodynamics: A Riemannian geometric model, Phys. Rev. A 20, 1608 (1979).

[23] P. Salamon and R. S. Berry, Thermodynamic Length and Dissipated Availability, Phys. Rev. Lett. 51, 1127 (1983).

[24] S. Deffner and E. Lutz, Generalized Clausius Inequality for Nonequilibrium Quantum Processes, Phys. Rev. Lett. 105, 170402 (2010).

[25] E. A. Morozova and N. N. Cencov, Markov invariant geometry on manifolds of states, J. Sov. Math. 56, 2648 (1991).

[26] D. Petz and H. Hasegawa, On the Riemannian metric of entropies of density matrices, Lett. Math. Phys. 38, 221 (1996)

[27] D. P. Pires, M. Cianciaruso, L. C. Céleri, G. Adesso, and D. O. Soares-Pinto, Generalized Geometric Quantum Speed Limits, Phys. Rev. X 6, 021031 (2016).

[28] S. Luo, Wigner-Yanase skew information vs quantum Fisher information, Proc. Am. Math. Soc. 132, 885 (2004).

[29] L. Mancino, M. Sbroscia, I. Gianani, E. Roccia, and M. Barbieri, Quantum Simulation of Single-Qubit Thermometry Using Linear Optics, Phys. Rev. Lett. 118, 130502 (2017).

[30] W. K. Tham, H. Ferretti, A. V. Sadashivan, and A. M. Steinberg, Simulating and optimising quantum thermometry using single photons, Sci. Rep. 6, 38822 (2016).

[31] S. Jevtic, D. Newman, T. Rudolph, and T. M. Stace, Singlequbit thermometry, Phys. Rev. A 91, 012331 (2015).

[32] S. Campbell, M. G. Genoni, and S. Deffner, Precision thermometry and the quantum speed limit, Quantum Sci. Technol. 3, 025002 (2018).

[33] H.-P. Breuer and F. Petruccione, The Theory of Open Quantum Systems (Oxford University Press on Demand, New York, 2002).

[34] M. J. Donald, Free energy and the relative entropy, J. Stat. Phys. 49, 81 (1987).

[35] S. Deffner and E. Lutz, Nonequilibrium Entropy Production for Open Quantum Systems, Phys. Rev. Lett. 107, 140404 (2011).

[36] K. M. R. Audenaert and J. Eisert, Continuity bounds on the quantum relative entropy, J. Math. Phys. 46, 102104 (2005).

[37] N. N. Čencov, Statistical Decision Rules and Optimal Inference (American Mathematical Society, Providence, 1982), Vol. 53. 
[38] See Supplemental Material at http://link.aps.org/ supplemental/10.1103/PhysRevLett.121.160602 for a proof of the triangle inequality.

[39] M. A. Nielsen and I. L. Chuang, Quantum Computation and Quantum Information (Cambridge University Press, Cambridge, England, 2000).

[40] Energies are measured in units of the gap between the two levels of the Hamiltonian.

[41] D.-S. Wang, D. W. Berry, M. C. de Oliveira, and B.C. Sanders, Solovay-Kitaev Decomposition Strategy for Single-Qubit Channels, Phys. Rev. Lett. 111, 130504 (2013).

[42] H. Lu, C. Liu, D. Wang et al., Experimental quantum channel simulation, Phys. Rev. A 95, 042310 (2017).

[43] Á. Cuevas, A. Mari, A. De Pasquale, A. Orieux, M. Massaro, F. Sciarrino, P. Mataloni, and V. Giovannetti, Cut-and-paste restoration of entanglement transmission, Phys. Rev. A 96, 012314 (2017).

[44] Á. Cuevas, A. De Pasquale, A. Mari, A. Orieux, S. Duranti, M. Massaro, A. Di Carli, E. Roccia, J. Ferraz, F. Sciarrino, P. Mataloni, and V. Giovannetti, Amending entanglementbreaking channels via intermediate unitary operations, Phys. Rev. A 96, 022322 (2017).

[45] N. K. Langford, T. J. Weinhold, R. Prevedel, K. J. Resch, A. Gilchrist, J. L. O'Brien, G. J. Pryde, and A. G. White, Demonstration of a Simple Entangling Optical Gate and
Its Use in Bell-State Analysis, Phys. Rev. Lett. 95, 210504 (2005).

[46] N. Kiesel, C. Schmid, U. Weber, R. Ursin, and H. Weinfurter, Linear Optics Controlled-Phase Gate Made Simple, Phys. Rev. Lett. 95, 210505 (2005).

[47] K. Okamoto, H. F. Hofmann, S. Takeuchi, and K. Sasaki, Demonstration of an Optical Quantum Controlled-NOT Gate without Path Interference, Phys. Rev. Lett. 95, 210506 (2005).

[48] L. Mancino, M. Sbroscia, E. Roccia, I. Gianani, F. Somma, P. Mataloni, M. Paternostro, and M. Barbieri, The entropic cost of quantum generalized measurements, npj Quantum Inf. 4, 20 (2018).

[49] E. Roccia, I. Gianani, L. Mancino, M. Sbroscia, F. Somma, M. G. Genoni, and M. Barbieri, Entangling measurements for multiparameter estimation with two qubits, Quantum Sci. Technol. 3, 01LT01 (2018).

[50] M. S. Palsson, M. Gu, J. Ho, H. M. Wiseman, and G. J. Pryde, Experimentally modeling stochastic processes with less memory by the use of a quantum processor, Sci. Adv. 3, e1601302 (2017).

[51] E. Lubkin, Keeping the entropy of measurement: Szilard revisited, Int. J. Theor. Phys. 26, 523 (1987).

[52] V. Vedral, The role of relative entropy in quantum information theory, Rev. Mod. Phys. 74, 197 (2002). 